PROCEEDINGS OF THE

AMERICAN MATHEMATICAL SOCIETY

Volume 129, Number 6, Pages 1799-1803

S 0002-9939(00)05730-0

Article electronically published on November 21, 2000

\title{
THE ABELIANIZATION OF ALMOST FREE GROUPS
}

\author{
CHARLY BITTON \\ (Communicated by Carl G. Jockusch, Jr.)
}

\begin{abstract}
We will construct an almost free non-free group $G$ of cardinality
$\aleph_{1}$ such that $G / G^{\prime}$ is a free abelian group.
\end{abstract}

\section{INTRODUCTION}

In this paper, we will deal with almost free non-free groups and almost free nonfree abelian groups. A group $G$ of cardinality $\lambda$ is said to be almost free if every subgroup of $G$ of cardinality less than $\lambda$ is free. Similarly, an abelian group $A$ of cardinality $\lambda$ is said to be almost free if every subgroup of $A$ of cardinality less than $\lambda$ is free abelian. In 1951, Higman [2] constructed an almost free non-free group of cardinality $\aleph_{1}$; since then, almost free groups and almost free abelian groups have been investigated by a number of authors. In particular, Shelah [6] proved that if there exists an almost free non-free abelian group of cardinality $\lambda$, then there also exists an almost free non-free group of cardinality $\lambda$. The converse of this result is still open and only partial results are known in this direction (see [1]). There is a natural way to approach this question. Suppose that $G$ is an almost free non-free group of cardinality $\lambda$. Then an easy argument shows that the quotient group $G / G^{\prime}$ is an almost free abelian group of cardinality $\lambda$. (Here $G^{\prime}$ denotes the commutator subgroup of $G$.) If it could be shown that $G / G^{\prime}$ is necessarily non-free, then the problem would be solved. In this paper, we will construct an almost free non-free group $G$ such that $G / G^{\prime}$ is a free abelian group. Consequently, this naive approach to the problem cannot succeed.

\section{The ABELIANIZATION OF ALMOST FREE NON-FREE GROUPS}

In this section, we will construct an almost free non-free group $G$ of cardinality $\aleph_{1}$ such that $G / G^{\prime}$ is a free abelian group of cardinality $\aleph_{1}$.

Before starting the proof, we will review some group theoretic results and establish our notation. Suppose that $G$ is a free group. Then it is well known that every subgroup $H$ of $G$ is free. We will write $H \mid G$ if $H$ is a free factor of $G$; i.e., if there exists a basis $X$ of $G$ and a subset $X^{\prime} \subseteq X$ such that $X^{\prime}$ is a basis of $H$. In this case, if $E$ is the subgroup generated by $X \backslash X^{\prime}$, then $G=H * E$ is the free product of $H$ and $E$. If $Y$ is any subset of $G$, then $\langle Y\rangle$ denotes the subgroup of $G$ which is

Received by the editors May 5, 1999 and, in revised form, October 5, 1999.

1991 Mathematics Subject Classification. Primary 03E75, 03E05, 20 K27.

This is part of the author's Ph.D. thesis done under the supervision of Professor M. Magidor to whom the author is greatly indebted for his help. 
generated by $Y$. If $H$ is any subgroup of $G$, then $H^{G}$ denotes the normal closure of $H$ in $G$; i.e., the smallest normal subgroup of $G$ which contains $H$.

We will make repeated use of the following result.

Lemma 2.1. Suppose that $G$ is a free group and that $K \mid G$. If $H$ is any subgroup of $G$, then $H \cap K \mid H$.

Proof. For example, see Problem 32 in Section 2.4 of [4].

We will also make use of the following characterization of the commutator subgroup of a free group.

Lemma 2.2. Suppose that the group $A$ is freely generated by the set $\mathcal{A}$. For each $x \in \mathcal{A}$, let $\ell_{x}^{A}: A \rightarrow \mathbb{Z}$ be the function such that $\ell_{x}^{A}(w)$ is the sum of the exponents of the occurrences of $x$ in the word $w \in A$. Then $w \in A^{\prime}$ iff $\ell_{x}^{A}(w)=0$ for every $x \in \mathcal{A}$.

Proof. For example, see Problem 2 in Section 2.2 of [4].

Definition 2.3. If $\kappa$ is an infinite cardinal, then we say that the principle $F(\kappa)$ holds iff there is a free group $A$ of rank $\kappa$ and a free subgroup $B$ of rank $\kappa$ such that:

(I) If $C$ is a free factor of $B$ of rank less than $\kappa$, then $C$ is also a free factor of $A$.

(II) $B$ is not a free factor of $A$.

(III) $B \cap A^{\prime}=B^{\prime}$.

In 2, Higman used a pair of free groups of rank $\aleph_{0}$ satisfying conditions (I) and (II) of principle $F\left(\aleph_{0}\right)$ to construct an almost free non-free group of cardinality $\aleph_{1}$. We will essentially give his construction in the proof of Lemma 2.5. We have added condition (III) in order to ensure that $B / B^{\prime}$ is naturally embedded into $A / A^{\prime}$ via the map $b B^{\prime} \mapsto b A^{\prime}$. Later we will construct a pair of free groups $(B, A)$ satisfying the conditions of principle $F\left(\aleph_{0}\right)$ in such a way that the natural embedding $B / B^{\prime} \hookrightarrow$ $A / A^{\prime}$ is surjective. In this case, we will write $B / B^{\prime}=A / A^{\prime}$.

Theorem 2.4. There exists an almost free non-free group $G$ of cardinality $\aleph_{1}$ such that $G / G^{\prime}$ is a free abelian group of cardinality $\aleph_{1}$.

We will break the proof of Theorem 2.4 into a series of lemmas. We will use the next lemma to construct the group $G$ from a suitably chosen pair $(A, B)$ of free groups exemplifying principle $F\left(\aleph_{0}\right)$.

Lemma 2.5. Principle $F\left(\aleph_{0}\right)$ implies that there exists an almost free non-free group $G$ of cardinality $\aleph_{1}$.

Proof. Suppose that the free groups $B \leqslant A$ exemplify principle $F\left(\aleph_{0}\right)$. We will define a smooth strictly increasing chain of free group $G_{\alpha}$ by induction on $\alpha<\aleph_{1}$. Let $G_{0}$ be a free group of rank $\aleph_{0}$. Now suppose inductively that $\alpha>0$ and that $G_{\beta}$ has been defined for all $\beta<\alpha$. Suppose further that if $\gamma<\beta<\alpha$, then every finitely generated free factor of $G_{\gamma}$ is also a free factor of $G_{\beta}$. First suppose that $\alpha$ has the form $\beta+2$. In this case, we let $G_{\alpha}=G_{\beta+1} *\left\langle x_{\beta+1}\right\rangle$, where $\left\langle x_{\beta+1}\right\rangle$ is an infinite cyclic group such that $G_{\beta+1} \cap\left\langle x_{\beta+1}\right\rangle=\{e\}$. Next suppose that $\alpha=\delta+1$ for some limit ordinal $\delta$. Then we let $G_{\alpha}$ be a free group such that $G_{\delta} \leqslant G_{\alpha}$ and 
the following diagram commutes:

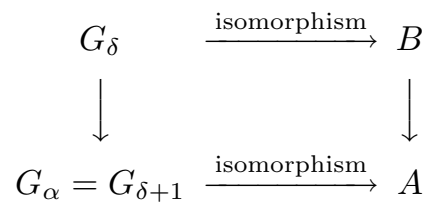

Finally if $\alpha$ is a limit ordinal, then we let $G_{\alpha}=\bigcup_{\beta<\alpha} G_{\beta}$. In this case, it is necessary to check that $G_{\alpha}$ is a free group. To see this, first let $\left\langle g_{i} \mid i<\omega\right\rangle$ be an enumeration of the elements of $G_{\alpha}$; then choose inductively an increasing sequence of ordinals

$$
\beta_{0} \leq \beta_{1} \leq \cdots \leq \beta_{n} \leq \cdots<\alpha
$$

and an increasing sequence of subgroups of $G_{\alpha}$

$$
F_{0} \leqslant F_{1} \leqslant \cdots \leqslant F_{n} \leqslant \cdots
$$

such that $\left\{g_{0}, \ldots, g_{n}\right\} \subseteq F_{n}$ and $F_{n}$ is a finitely generated free factor of $G_{\beta_{n}}$. By Lemma [2.1, $F_{n}$ is a free factor of $F_{n+1}$ for each $n<\omega$. Since $G_{\alpha}=\bigcup_{n<\omega} F_{n}$, it follows that $G_{\alpha}$ is a free group.

Now let $G=\bigcup_{\alpha<\aleph_{1}} G_{\alpha}$. Then it is clear that $G$ is almost free. Suppose that $G$ is free. Then we can express $G=\bigcup_{\alpha<\aleph_{1}} K_{\alpha}$ as the union of a smooth strictly increasing chain of countable subgroups such that $K_{\alpha} \mid G$ for all $\alpha<\aleph_{1}$. There exists a club $C$ such that $K_{\gamma}=G_{\gamma}$ for all $\gamma \in C$; so by Lemma 2.1, $G_{\gamma} \mid G_{\gamma+1}$ for all $\gamma \in C$. But by taking $\gamma \in C$ to be a limit ordinal, we obtain a contradiction.

Lemma 2.6. In the above construction, $G_{\alpha} \cap G^{\prime}=G_{\alpha}^{\prime}$.

Proof. An easy induction shows that if $\alpha<\beta$, then $G_{\alpha} \cap G_{\beta}^{\prime}=G_{\alpha}^{\prime}$. (The only point where the induction could conceivably break down is when $\alpha$ is a limit ordinal and $\beta=\alpha+1$. However, condition (III) of principle $F\left(\aleph_{0}\right)$ deals with this case.) The result follows.

Now we want to define a particular pair of free groups $(B, A)$ exemplifying principle $F\left(\aleph_{0}\right)$ such that if $G$ is the group constructed in Lemma 2.5, then $G / G^{\prime}$ is a free abelian group of cardinality $\aleph_{1}$. We shall make use of the following result of Levi. Recall that if $H$ is any group, then the $n^{\text {th }}$ derived subgroup $H^{(n)}$ of $H$ is defined inductively by $H^{(0)}=H$ and $H^{(n+1)}=\left(H^{(n)}\right)^{\prime}$.

Lemma 2.7. If $H$ is a free group, then $\bigcap_{n<\omega} H^{(n)}=\{e\}$.

Proof. This is an immediate consequence of Corollary 2.12 of [4].

Definition 2.8. Let $A$ be the group freely generated by the set $\left\{a_{n} \mid n \geq 1\right\}$ and let

$$
B=\left\langle a_{1}^{-1}\left[a_{2}, a_{3}\right], a_{2}^{-1}\left[a_{3}, a_{4}\right], a_{3}^{-1}\left[a_{4}, a_{5}\right], \ldots, a_{n}^{-1}\left[a_{n+1}, a_{n+2}\right], \ldots\right\rangle .
$$

Lemma 2.9. (1) Every finitely generated free factor of $B$ is also a free factor of A.

(2) $B$ is not a free factor of $A$.

(3) $B \cap A^{\prime}=B^{\prime}$.

(4) $B / B^{\prime}=A / A^{\prime}$. 
Proof. First we will show that every finitely generated free factor of $B$ is also a free factor of $A$. For each $n>2$, define $A_{n}=\left\langle a_{1}, \ldots, a_{n}\right\rangle$ and

$$
B_{n}=\left\langle a_{1}^{-1}\left[a_{2}, a_{3}\right], a_{2}^{-1}\left[a_{3}, a_{4}\right], \ldots, a_{n-2}^{-1}\left[a_{n-1}, a_{n}\right]\right\rangle .
$$

Then it is easily checked that $A_{n}=B_{n} *\left\langle a_{n-1}, a_{n}\right\rangle$. Hence $B_{n}$ is a free factor of $A$. If $C$ is a finitely generated free factor of $B$, then there exists an integer $n>2$ such that $C \leqslant B_{n}$. By Lemma 2.1 $C$ is a free factor of $B_{n}$. Since $B_{n}$ is a free factor of $A$, it follows that $C$ is also a free factor of $A$.

Next we will prove that $B$ is not a free factor of $A$. To prove this, it is enough to show that $H=A / B^{A}$ is not a free group. (Recall that $B^{A}$ denotes the normal closure of $B$ in $A$.) Note that $H$ is generated by the elements $\left\{a_{n} \mid n \geq 1\right\}$ subject to the relations

$$
a_{1}=\left[a_{2}, a_{3}\right], a_{2}=\left[a_{3}, a_{4}\right], a_{3}=\left[a_{4}, a_{5}\right], \ldots, a_{n}=\left[a_{n+1}, a_{n+2}\right], \cdots .
$$

So we see that

$$
a_{1}=\left[a_{2}, a_{3}\right] \in H^{(1)}
$$

and then that

$$
a_{1}=\left[a_{2}, a_{3}\right]=\left[\left[a_{3}, a_{4}\right],\left[a_{4}, a_{5}\right]\right] \in H^{(2)} .
$$

Continuing in this fashion, we see that $a_{1} \in \bigcap_{n<\omega} H^{(n)}$. It is easily seen that $H$ is isomorphic to the direct limit of the system

$$
H_{2} \stackrel{h_{2}}{\longrightarrow} H_{3} \stackrel{h_{3}}{\longrightarrow} \ldots \stackrel{h_{n-1}}{\longrightarrow} H_{n} \stackrel{h_{n}}{\longrightarrow} H_{n+1} \stackrel{h_{n+1}}{\longrightarrow} \ldots
$$

where $H_{n}$ is the free group $\left\langle a_{n-1}, a_{n}\right\rangle$ and $h_{n}: H_{n} \rightarrow H_{n+1}$ is the embedding such that $a_{n-1} \mapsto\left[a_{n}, a_{n+1}\right]$ and $a_{n} \mapsto a_{n}$. The element $a_{1} \in H$ corresponds to the constant sequence $\left(a_{1}, a_{1}, \cdots\right)$ in the direct limit and so $a_{1}$ is a nonidentity element of $H$. By Lemma 2.7, $H$ is not a free group.

Finally consider the canonical homomorphism $\pi: B \rightarrow A / A^{\prime}$ such that for each $n \geq 1$,

$$
\pi\left(a_{n}^{-1}\left[a_{n+1}, a_{n+2}\right]\right)=a_{n}^{-1}\left[a_{n+1}, a_{n+2}\right] A^{\prime}=a_{n}^{-1} A^{\prime} .
$$

Clearly $\pi$ is surjective and $\operatorname{ker} \pi=B \cap A^{\prime}$. Note that

$$
\begin{aligned}
w \in \operatorname{ker} \pi & \text { iff } \ell_{x}^{A}(w)=0 \text { for all } x \in\left\{a_{n} \mid n \geq 1\right\} \\
& \text { iff } \ell_{y}^{B}(w)=0 \text { for all } y \in\left\{a_{n}^{-1}\left[a_{n+1}, a_{n+2}\right] \mid n \geq 1\right\}, \\
& \text { iff } w \in B^{\prime} .
\end{aligned}
$$

(The last equivalence is a consequence of Lemma 2.2]) Thus $B \cap A^{\prime}=B^{\prime}$ and $B / B^{\prime}=A / A^{\prime}$.

Lemma 2.10. $G / G^{\prime}$ is free abelian of cardinality $\aleph_{1}$.

Proof. For each $\alpha<\aleph_{1}$, let $A_{\alpha}=G_{\alpha} / G_{\alpha}^{\prime}$. By Lemma 2.6, we have that

$$
\begin{aligned}
G / G^{\prime} & =\bigcup_{\alpha<\aleph_{1}} G_{\alpha} / G_{\alpha} \cap G^{\prime} \\
& =\bigcup_{\alpha<\aleph_{1}} G_{\alpha} / G_{\alpha}^{\prime}=\bigcup_{\alpha<\aleph_{1}} A_{\alpha} .
\end{aligned}
$$

If $\alpha$ is a limit ordinal, then $G_{\alpha} / G_{\alpha}^{\prime}=G_{\alpha+1} / G_{\alpha+1}^{\prime}$ and so $A_{\alpha}=A_{\alpha+1}$. On the other hand, if $\alpha$ is a successor ordinal, then $A_{\alpha+1}=A_{\alpha} \oplus\left\langle\bar{x}_{\alpha}\right\rangle$, where $\bar{x}_{\alpha}$ denotes 
the image of the element $x_{\alpha} \in G_{\alpha+1}$ in the quotient group $A_{\alpha+1}$. It follows that if $\alpha<\beta$, then $A_{\alpha}$ is a direct summand of $A_{\beta}$. It is now clear that $G / G^{\prime}$ is a free abelian group of cardinality $\aleph_{1}$.

This completes the proof of Theorem 2.4

\section{Concluding REMARKS}

Finally we will say a few words about extending our main result to cardinalities $\lambda>\aleph_{1}$. We need only consider regular cardinals $\lambda$, since Shelah [5] has proved that if $G$ is an almost free group of singular cardinality, then $G$ is free. (The corresponding result is also true for almost free abelian groups of singular cardinality.) In [6], Shelah discovered that the purely combinatorial principle $N P T(\lambda)$ is equivalent to the existence of an almost free non-free abelian group; in [3], it was shown in $Z F C$ that $N P T(\lambda)$ holds for an unbounded set of cardinals below the first cardinal fixed point. If $N P T(\lambda)$ holds, then it is possible to generalize the proof of Theorem 2.4 and construct an almost free non-free group $G$ of cardinality $\lambda$ such that $G / G^{\prime}$ is a free abelian group. Of course, the real problem is to produce such a group when $N P T(\lambda)$ fails.

\section{ACKNOWLEDGEMENT}

The author wishes to express his appreciation to the referee for making many improvements and helpful comments.

\section{REFERENCES}

[1] C. Bitton, Problems in set theory arising from group theory, PhD thesis, The Hebrew University, 1998

[2] G. Higman, Almost free groups, Proc. London Math. Soc. (3) 1 (1951), 284-290. MR 13:430d

[3] M. Magidor and S. Shelah, When does almost free imply free?, J. Amer. Math. Soc. 7 (1994), 769-830. MR 94m:03081

[4] W. Magnus, A. Karrass and D. Solitar, Combinatorial Group Theory, Dover Publications, New York, 1966. MR 34:7617

[5] S. Shelah, A compactness theorem for singular cardinals, free algebras, Whitehead problem and transversals, Israel J. Math 21 (1975), 319-349. MR 52:10410

[6] S. Shelah, Incompactness in regular cardinals, Notre Dame J. Formal Logic 26 (1985), 195228. MR 87f:03095

Institute of Mathematics, Hebrew University, Jerusalem, Israel

Current address: Department of Mathematics, University of California, Irvine, California 92679

E-mail address: cbitton@math.uci.edu 